\title{
EROS 2 proper motion survey: Constraints on the halo white dwarfs ${ }^{\star}$
}

\author{
B. Goldman ${ }^{1,2,3}$, C. Afonso ${ }^{1,2,3}$, Ch. Alard ${ }^{4}$, J.-N. Albert ${ }^{5}$, A. Amadon ${ }^{1}$, J. Andersen ${ }^{6}$, R. Ansari ${ }^{5}$,
} É. Aubourg ${ }^{1}$, P. Bareyre ${ }^{1,2}$, F. Bauer ${ }^{1}$, J.-Ph. Beaulieu ${ }^{7}$, G. Blanc ${ }^{1}$, A. Bouquet ${ }^{2}$, S. Char ${ }^{\dagger}$, X. Charlot ${ }^{1}$,

F. Couchot ${ }^{5}$, Ch. Coutures ${ }^{1}$, F. Derue ${ }^{1}$, R. Ferlet ${ }^{7}$, P. Fouqué ${ }^{8,13}$, J.-F. Glicenstein ${ }^{1}$, A. Gould ${ }^{2,9}$,

D. Graff ${ }^{12}$, M. Gros ${ }^{1}$, J. Haïssinski ${ }^{5}$, C. Hamadache ${ }^{1}$, J.-Ch. Hamilton ${ }^{2}$, D. Hardin ${ }^{10}$, J. de Kat ${ }^{1}$, A. Kim ${ }^{1, \star \star}$, Th. Lasserre ${ }^{1}$, L. Le Guillou ${ }^{1}$, É. Lesquoy ${ }^{1,7}$, C. Loup ${ }^{7}$, Ch. Magneville ${ }^{1}$, B. Mansoux ${ }^{5}$, J.-B. Marquette ${ }^{7}$, É. Maurice ${ }^{11}$, A. Maury ${ }^{13}$, A. Milsztajn ${ }^{1}$, M. Moniez $^{5}$, N. Palanque-Delabrouille ${ }^{1}$, O. Perdereau ${ }^{5}$, L. Prévot ${ }^{11}$, N. Regnault ${ }^{5, \star \star}$, J. Rich ${ }^{1}$, M. Spiro ${ }^{1}$, P. Tisserand ${ }^{1}$, A. Vidal-Madjar ${ }^{7}$, L. Vigroux ${ }^{1}$, and S. Zylberajch ${ }^{1}-$ The ERos collaboration

1 CEA, DSM, DAPNIA, Centre d'Études de Saclay, 91191 Gif-sur-Yvette Cedex, France

2 Collège de France, Physique Corpusculaire et Cosmologie, IN2P3 CNRS, 11 pl. M. Berthelot, 75231 Paris Cedex, France

3 NMSU, Department of Astronomy, Las Cruces, New Mexico 88003, USA

${ }^{4}$ DASGAL, 77 avenue de l'Observatoire, 75014 Paris, France

5 Laboratoire de l'Accélérateur Linéaire, IN2P3 CNRS, Université Paris-Sud, 91405 Orsay Cedex, France

6 Astronomical Observatory, Copenhagen University, Juliane Maries Vej 30, 2100 Copenhagen, Denmark

7 Institut d'Astrophysique de Paris, INSU CNRS, 98bis boulevard Arago, 75014 Paris, France

8 LESIA, Observatoire de Meudon, 92195 Meudon Cedex, France

9 Ohio State University, Department of Astronomy, Columbus, OH 43210, USA

10 LPNHE, IN2P3-CNRS-Universités Paris VI et VII, 4 place Jussieu, 75252 Paris Cedex 05, France

11 Observatoire de Marseille, 2 pl. Le Verrier, 13248 Marseille Cedex 04, France

12 University of Michigan, Department of Astronomy, Ann Arbor, MI 48109, USA

13 ESO, Casilla 19001, Santiago 19, Chile

Received 4 March 2002 / Accepted 3 June 2002

\begin{abstract}
We are conducting a $377^{\circ 2}$ proper motion survey in the $\sim V$ and $I$ bands in order to determine the cool white dwarf contribution to the Galactic dark matter. Using the $250^{\circ 2}$ for which we possess three epochs, and applying selection criteria designed to isolate halo-type objects, we find no candidates in a $5500 \mathrm{pc}^{3}$ effective volume for old, fast $M_{V}=17$ white dwarfs. We check the detection efficiency by cross-matching our catalogue with Luyten's NLTT catalogue. The halo white dwarf contribution cannot exceed $5 \%$ (95\% C.L.) for objects with $M_{V}=17$ and $1 \leq V-I \leq 1.5$. The same conclusion applies to a 14 Gyr halo composed of white dwarfs with hydrogen atmosphere, as modeled by Chabrier.
\end{abstract}

Key words. Galaxy: halo - Galaxy: kinematics and dynamics - dark matter - white dwarfs

\section{Introduction}

The rotation of the Milky Way is believed to be sustained by a massive dark halo, at least beyond one disk scale length. Old, cool white dwarfs (WDs) are one of the proposed constituents of that dark matter. A number of constraints have been placed on the total amount of halo

Send offprint requests to: B.Goldman,

e-mail: bgoldman@nmsu.edu.

* Based on observations made with the Marly telescope, located at the European Southern Observatory, La Silla, Chile.

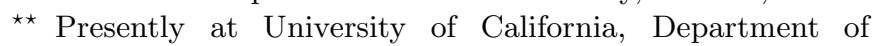
Physics, Berkeley, CA 97720, USA. white dwarfs (HWDs). One is based on the light emitted by WD progenitors (Graff et al. 1999). Extragalactic metallicity measurements also place strong constraints through the metal and helium enrichment due to the HWDs (Freese 2000), although mechanisms have been proposed to evade those constraints.

Over the past three years a great excitement has come from proper motion surveys used to find fast WDs in the Solar neighbourhood. The surveys conducted by Luyten (1979a) and Knox et al. (1999) are jointly sensitive to $1500 \mathrm{pc}^{3}$ for $M_{V}=17.5$ HWDs (Flynn et al. 2001). They find no halo WD (Liebert et al. 1988), although this is 
disputed in the case of Luyten's survey, whose efficiency is still a matter of strong debate, 23 years after publication. On the other hand, several collaborations have reported proper motion detection of fast, cool WDs some of them being subsequently confirmed spectroscopically (Ibata et al. 2000; Oppenheimer et al. 2001a). Nelson et al. (2002) also reported the discovery of new HWD candidates in the HST Groth-Westphal strip, although using only two epochs. Finally, Monet et al. (2000) discovered new high velocity WDs but the small sample of new objects confirms the high efficiency of Luyten (1979b), thus decreasing the maximum density of halo WDs.

On the microlensing side, Lasserre et al. (2000) put a $30 \%$ upper limit (95\% C.L.) on the contribution of HWDs of any age - or any compact object of similar mass - to a standard halo, while Alcock et al. (2000) interpret their 13 microlensing candidates towards the Large Magellanic Cloud as $0.15-0.9 M_{\odot}$ objects contributing $20_{-6}^{+11} \%$ to the standard halo.

The interesting controversy developed around the Oppenheimer et al. (2001b) results addresses the question of the nature of their findings: thick disk or halo objects. It is in any case agreed that these relatively bright $M_{I} \approx 14$, young WDs contribute very little to the Galactic dark matter, a few percent at most. It has been suggested however that these could be the bright tail of a fainter, older population (Hansen 2001).

Our proper motion survey began in 1996 using the EROS 2 cameras. It was designed to study the contribution of older, fainter WDs to the halo, and also addresses the question raised by Hansen (2001). Like other proper motion searches, ours takes advantage of the large expected velocity dispersion of halo objects, and it is not intended to address the question of the low velocity, or brighter, WDs that most recent surveys probe (Oppenheimer et al. 2001a; Majewski \& Siegel 2002). Here we report our results based on the analysis of $250^{\circ 2}$ that were observed during at least three epochs. These yield a sensitivity about three times that of Luyten (1979b), depending on HWD colours and luminosities.

\section{The EROS 2 proper motion survey}

\subsection{Instrument and observations}

The ERos 2 wide-field imager (Bauer \& de Kat 1998) was designed to search for microlensing effects towards the Galactic bulge and disk and the Magellanic Clouds. Its two $1^{\circ 2}$ CCD cameras are mounted at the Cassegrain focus of the 1-m Marly telescope at La Silla (Chile), with a pixel size of $0^{\prime \prime} 6$. These two $8 \mathrm{k} \times 4 \mathrm{k}$ mosaics are illuminated through a dichroic beam splitter, which, together with the CCD efficiency, defines the bandpasses. The colour transformations between the EROs system $\left(\mathcal{V}_{\mathrm{E}}, \mathcal{I}_{\mathrm{E}}\right)$ and the standard Johnson-Cousins $(V, I)$ system are determined by observing Landolt (1992) standards and Ogle (Paczynski et al. 1999) secondary standards in the Baade window: $\mathcal{V}_{\mathrm{E}}=V-(0.32 \pm 0.03)(V-I)$ and $\mathcal{I}_{\mathrm{E}}=I+(0.06 \pm 0.03)(V-I)$. We estimate the precision of the colour transformations to be $0.1^{\text {mag }}$ for $V-I=0-2$, the range of expected colours for HWDs. Comparison between our $\mathcal{I}_{\mathrm{E}}$ photometry and $I$ as measured by DENIS (Fouqué et al. 2000) reveals a small offset, $I_{\text {Eros }}=I_{\text {Denis }}-0.06$, while a comparison between our photometry and the Photometric GSC (Lasker et al. 1988) reveals no significant offset, with a $0.05^{\mathrm{mag}}$ uncertainty.

Proper motion observations were performed one to two hours per dark night, within $90 \mathrm{~min}$. of the meridian to minimize atmospheric refraction. Our limiting magnitudes are about $V=21.5$ and $I=20.5$. We observed $190^{\circ 2}$ in the Southern Galactic Hemisphere $\left(-79^{\circ}<b_{\text {gal }}<-48^{\circ}\right)$, in the following strips along the $\alpha$ coordinate, $\Delta \delta=1.4 \mathrm{deg}$ wide: $22 \mathrm{~h} 16 \mathrm{~min}<\alpha<$ $3 \mathrm{~h} 44 \mathrm{~min}$ at $\delta=-44^{\circ} 45 \mathrm{~min}, 23 \mathrm{~h} 31 \mathrm{~min}<\alpha<1 \mathrm{~h} 34 \mathrm{~min}$ at $\delta=-40^{\circ} 09 \mathrm{~min}$ and $22 \mathrm{~h} 24 \mathrm{~min}<\alpha \leq 3 \mathrm{~h} 28 \mathrm{~min}$ at $\delta=-38^{\circ} 45 \mathrm{~min}$, and $187^{\circ 2}$ in the Northern Hemisphere $\left(41^{\circ}<b_{\text {gal }}<59^{\circ}\right)$ with $10 \mathrm{~h} 57 \mathrm{~min}<\alpha \leq 13 \mathrm{~h} 23 \mathrm{~min}$ at $\delta=-12^{\circ}$ and $10 \mathrm{~h} 57 \mathrm{~min}<\alpha<12 \mathrm{~h} 53 \mathrm{~min}$ at $\delta=-4^{\circ} 36 \mathrm{~min}$.

Only those fields $\left(250^{\circ 2}\right)$ with three epochs separated by one-year intervals (two-year total baseline), in one or two bands, are taken into account in this letter.

\subsection{Proper motion catalogue}

The reduction software for source detection, classification and catalogue matching was written in the framework of the Eros PEIDA++ package (Ansari 1996). Since photon noise dominates the astrometric errors for most of the search volume, we use a simple two-dimensional Gaussian PSF-fitting algorithm to determine stellar positions. A rough star/galaxy classification is performed to limit galaxy contamination, with cuts chosen so that few stars are misclassified. The catalogues of the three epochs are geometrically aligned, with the deepest one taken as reference, using a linear transformation fitted to the 40 brightest stars, and matched within a search radius corresponding to a $6^{\prime \prime} \mathrm{yr}^{-1}$ proper motion. The RMS distance between matched stars provides an upper limit to the total astrometric error, which is 25 mas $(1 \sigma)$ for bright objects, degrading to 150 mas for $V=21$ or $I=20$.

In order to minimize contamination by spurious candidates we require that all objects be observed at least three times, separated by one-year intervals. The proper motions are derived from linear motion fits. The $\chi^{2}$ distributions deviate from the expected ones only for confidence levels lower than $0.5 \%$, indicating the onset of systematic effects. The $4 \%$ of objects so affected, mostly poorly measured galaxies, stars close to CCD defects or visual binaries, are removed from the analysis. 


\section{Search for halo white dwarfs}

\subsection{Selection of halo candidates}

The choice of the criteria used to distinguish halo objects from the much more numerous disk and thick disk stars, and from extragalactic objects, is of prime importance. We apply two cuts: We first require that the halo candidates detected in the $\mathcal{V}_{\mathrm{E}}$ band exhibit a reduced proper motion, $H_{V}=V+5 \log \frac{\mu}{1^{\prime \prime} \mathrm{yr}^{-1}}+5=M_{V}+5 \log \frac{v_{\perp}}{1 \mathrm{~km} \mathrm{~s}^{-1}}-3.38>$ 22.5 , where $\mu$ is the proper motion and $v_{\perp}$ the tangential velocity, and that those detected in $\mathcal{I}_{\mathrm{E}}$ have $H_{I}>21.5$. This cut select candidates that are both fast and faint (see Fig. 1).

We then require a proper motion $\mu>0.7 \mathrm{yr}^{-1}$. This cut removes objects whose proper motion reality cannot be confirmed by examining our images. It affects our sensitivity, depending on the mean WD magnitude: $\mu_{\min }=0.7 \mathrm{yr}^{-1}$ corresponds to a transverse velocity of $v_{\perp}=\frac{60 \mathrm{pc}}{d} \times 200 \mathrm{~km} \mathrm{~s}^{-1}$ at distance $d$. A typical $200 \mathrm{~km} \mathrm{~s}^{-1}$ transverse velocity is expected for halo objects, and $d=60 \mathrm{pc}$ is the typical distance of WDs in our effective volume for $M_{V}=17$ stars. In fact, a detailed simulation shows that this cut reduces our sensitivity by $20 \%$ (resp. 45\%) for $M_{V}=18$ (resp. 17) HWDs.

The proper motion cut was set to $\mu>0.7 \mathrm{yr}^{-1}$ as a result of the following optimization: on the one hand this value is low enough to ensure a high detection efficiency of HWDs and, on the other hand, it is sufficiently high to eliminate candidates which are likely to be spurious because of a poor measurement of their proper motion. Indeed, when the proper motion cut is lowered to 0 .' $6 \mathrm{yr}^{-1}, 10$ candidates pass the cut, but for those whose proper motions were measured in both bands (6 among the 10 candidates) we find that for all of them the measured values of their visible and red proper motion are incompatible.

No candidates survive the two cuts: $H_{V}>22.5$ or $H_{I}>21.5$, and $\mu>0$.'7 $\mathrm{yr}^{-1}$. Had we required $\mu>$ 0 .' $8 \mathrm{yr}^{-1}$, our upper limits on the HWD contribution to the halo given below would move up by less than $1 \%$ of the standard dark halo.

\subsection{Comparison with Luyten's catalogue}

We cross match our proper motion catalogue with the NLTT catalogue (Luyten 1979b), by searching for bright stars in our catalogue with proper motion higher than 0 .' $1 \mathrm{yr}^{-1}$, within $1^{\prime}$ of Luyten positions. It turns out that $85 \%$ of Luyten's stars are recovered with compatible proper motion and proper motion angle, which demonstrates our high detection efficiency for stars similar to Luyten's stars. The $15 \%$ loss is partly due to the expected loss due to defects in the CCDs (4\%) and to pointing dispersion between epochs (4\%). Additionally, our proper motion measurements for Luyten's stars are significant at the $3 \sigma$ level in each band, and are compatible at the $2 \sigma$ level. This gives us confidence in our proper motion

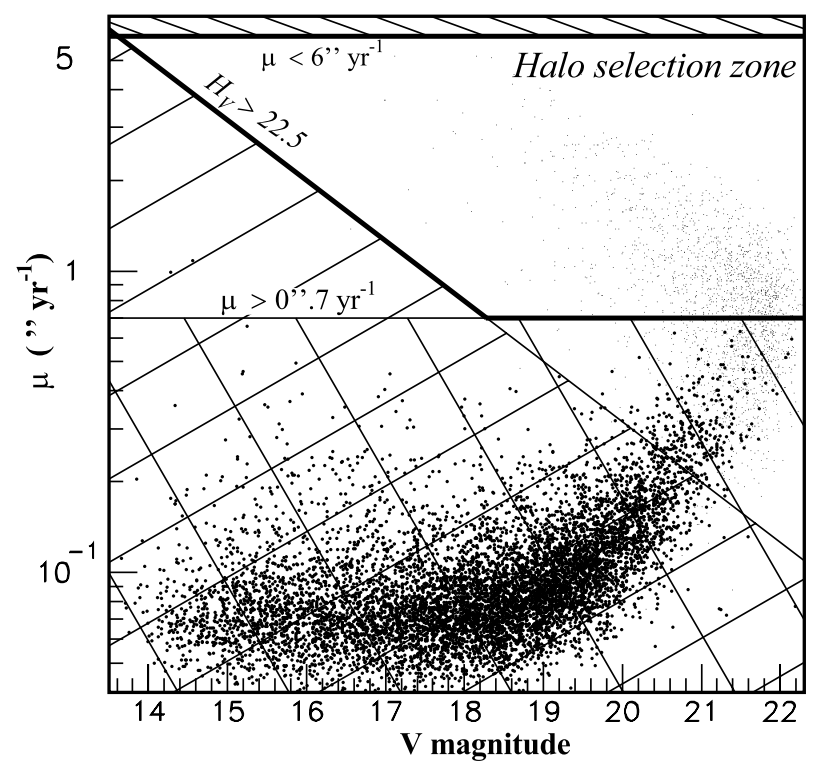

Fig. 1. Proper motion vs. $V$ magnitude for all objects detected in the $\mathcal{V}_{\mathrm{E}}$ band, whose proper motion significance is higher than $99.7 \%$ C.L. (big dots), and for a simulated isotropic standard halo of $M_{V}=17 \mathrm{WDs}$ fully composed of $0.6 M_{\odot}$ white dwarfs (small dots, statistics $\times 10$ ). The selection cuts exclude the hatched area. Here $45 \%$ of the HWDs are lost due to the $\mu>0.17 \mathrm{yr}^{-1}$ cut.

accuracy, as most Luyten stars have proper motion much lower than our $0 . \prime 7 \mathrm{yr}^{-1}$ halo selection cut. However, this only applies to bright stars, as there are no Luyten stars close to our photometric detection limit. Unfortunately we can draw no conclusion regarding Luyten's completeness based on this study, as we lack a reliable estimate of the contamination of our disk-like proper motion catalogue. This study will be reported in more detail in a forthcoming article.

\section{Discussion}

\subsection{Halo model predictions}

In order to check our sensitivity to HWDs, we perform a full simulation of our observations. We measure the detection efficiency of a star on each frame by adding simulated stars to real images. Using the Besançon model of the Galaxy (Robin et al. 2000), to which we add one HWD per field, we then construct a simulate catalogue for each of our fields and each of our images, and process these catalogues in the same way as our observations. The Besançon model is a consistent hydrodynamical description of Galactic star counts and proper motion, based on initial mass function and stellar evolution.

We use various halo models with different kinematics. We use isotropic velocity dispersions of $\sigma_{1 \mathrm{D}}=100,130$ or $156 \mathrm{~km} \mathrm{~s}^{-1}$, or anisotropic, spheroid-like dispersions, with $\sigma_{1 \mathrm{D}}=85,105$ or $125 \mathrm{~km} \mathrm{~s}^{-1}$ along the $V$ (Galactic rotation) direction. We suppose halo rotations of $-50,0$ or $50 \mathrm{~km} \mathrm{~s}^{-1}$. The expected number of detections depend on 
these parameters at the 5 to $10 \%$ level. For a given kinematics, flattened halos correspond to higher local densities, and thus to proportionately more detections.

We consider HWDs of $16.5 \leq M_{V} \leq 18$ and $-0.5 \leq$ $V-I \leq 1.5$, in steps of $0.5 \mathrm{mag}$. We also generate HWDs with hydrogen atmosphere according to the Chabrier (1999) luminosity functions for halo ages of 14 and 15 Gyr. The predicted number of detections, in either the $\mathcal{V}_{\mathrm{E}}$ or the $\mathcal{I}_{\mathrm{E}}$ band, are reported in Table 1, for the standard isotropic, isothermal halo, which has a local density of $8 \times 10^{-3} M_{\odot} \mathrm{pc}^{-3}$. Statistical errors are $5 \%$ while systematic errors are estimated to be less than $25 \%$, the quadrature sum of errors in calibration (15\%), efficiencies (10\%) and parameters of the halo kinematics (15\%).

Table 1. Number of detected WDs, in either $\mathcal{V}_{\mathrm{E}}$ or $\mathcal{I}_{\mathrm{E}}$ band, predicted for a standard, isotropic halo with a local HWD mass density of $8 \times 10^{-3} M_{\odot} \mathrm{pc}^{-3}$, and for $0.6 M_{\odot}$ HWDs of various colours and magnitudes, over $250^{\circ 2}$. The effective volume is given between parenthesis for $M_{V}=17$ in units of $10^{3} \mathrm{pc}^{3}$.

\begin{tabular}{|c|r|rr|r|r|}
\hline \hline$M_{V}$ & 16.5 & \multicolumn{2}{|c|}{17} & 17.5 & 18 \\
\hline-0.5 & 48.2 & 35.9 & $(2.69)$ & 16.7 & 9.8 \\
0. & 69.2 & 38.2 & $(2.87)$ & 24.5 & 14.7 \\
+0.5 & 83.9 & 46.5 & $(3.49)$ & 30.9 & 17.3 \\
1. & 106.5 & 74.1 & $(5.56)$ & 40.0 & 21.4 \\
1.5 & 140.6 & 111.3 & $(8.35)$ & 58.7 & 29.2 \\
\hline
\end{tabular}

We have not taken binarity effects into account. Double stars of similar magnitude would appear brighter than individual WDs, so that objects at the brighter, slower end of the distribution could be lost by the reduced proper motion cut. Furthermore, the number of systems to which we are sensitive would be smaller than the actual number of stars; our constraints would have to be relaxed accordingly.

\subsection{Constraints on the halo WD fraction and age}

The constraints on the contribution of WD to the halo are obtained from the above predictions and the lack of halo candidates, and shown in Fig. 2. We find that a 14 Gyr halo cannot be made of more than $5 \% 0.6$ or $1.0 M_{\odot}$ HWDs, at the $95 \%$ C.L., while a 15 Gyr halo cannot be made of more than $15 \%$ of $0.6 M_{\odot}$ HWDs, or $45 \%$ of $1.0 M_{\odot}$ HWDs.

\subsection{Comparison with other surveys}

Our results, based on a larger volume than the study by Flynn et al. (2001), confirm their conclusion. They are marginally compatible with results of Ibata et al. (2000), depending on the exact WD colours and absolute magnitudes.

Regarding Oppenheimer et al. (2001a), we do not exclude a 1 to $2 \%$ contribution by bright

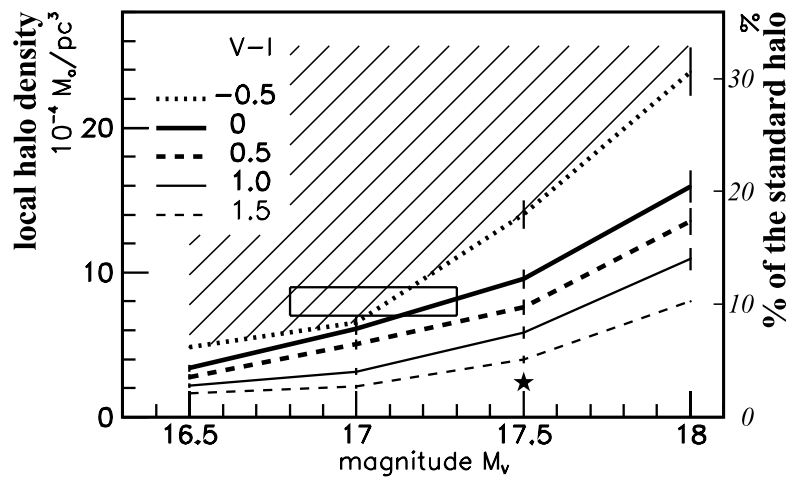

Fig. 2. Constraints on the WD contribution to the standard halo, for various WD colours, at the $95 \%$ C.L. The error bars correspond to the statistical error in the simulations. We exclude the hatched area. The empty box corresponds to the two WDs detected by Ibata et al. (2000), the star the result by Flynn et al. (2001) (no error bar given).

$M_{I}=13-15$ HWDs. We stress that our selection cut, $\mu>$ 0 .' $7 \mathrm{yr}^{-1}$, is more severe than theirs, while preserving most of the halo sensitivity for the faint magnitudes of interest here. Reid et al. (2001), Reylé et al. (2001) and Koopmans $\&$ Blandford (2002) also discuss this survey. We do constrain the proposition of (Hansen 2001) that the WDs detected by Oppenheimer et al. (2001a) be the bright tail of a fainter population. If this population has a halo-like kinematics, then the older part of the luminosity function, older by several Gyrs, cannot make up more than $\approx 7 \%$ of the halo.

Finally, our constraints are fully compatible with the ERos microlensing results. The constraints from the present study are even stronger than those from microlensing, for HWDs of absolute magnitude $M_{V}<18$ and also if the halo age is smaller than 15 Gyr (using the Chabrier 1999 luminosity function for DAs).

\section{Conclusion}

In this letter we have presented the analysis of $250^{\circ 2}$ of the ERos proper motion survey, dedicated to the search of old, faint white dwarfs. We found no halo white dwarf candidates. We exclude a white dwarf contribution to the halo above $5 \%$ for $M_{V}=17,1 \leq V-I \leq 1.5$, at the 95\% C.L. Assuming a Chabrier (1999) cooling model, a 14 Gyr halo cannot be made of more than $5 \%$ of WDs with hydrogen atmosphere. Our limits degrade to $20 \%$ for a 15 Gyr halo of $1 \mathrm{M}_{\odot}$ white dwarfs or for $M_{V}=18$, $0 \leq V-I \leq 1.5$ white dwarfs.

Acknowledgements. We thank Annie Robin and her collaborators for allowing us to use the latest version of the Besançon model prior to publication. We thank also Nicolas Epchtein and the DENIS collaboration for providing infrared unpublished photometry of our sources in order to check our $\mathcal{I}_{\mathrm{E}}$ calibration, and Gilles Chabrier for his comments. This research has made use of the SIMBAD database, operated at CDS, Strasbourg, France. We are grateful to Daniel Lacroix and the technical staff at the Observatoire de Haute Provence and to 
André Baranne for their help in refurbishing the MARLY telescope and remounting it in La Silla. We also thank the technical staff of ESO La Silla for their support of ERos. We thank Jean-François Lecointe and Adelino Gomes for assistance with the online computing. Work by A.G. was supported by NSF grant AST 97-27520 and by a grant from le Centre français pour l'accueil et les échanges internationaux.

\section{References}

Alcock, C., Allsman, R. A., Alves, D. R., et al. 2000, ApJ, 542, 281

Ansari, R. 1996, Vistas in Astron., 40, 519

Bauer, F., \& de Kat, J. 1998, in Optical Detectors for Astronomy, 191

Chabrier, G. 1999, ApJ, 513, L103

Flynn, C., Sommer-Larsen, J., Fuchs, B., Graff, D. S., \& Salim, S. 2001, MNRAS, 322, 553

Fouqué, P., Chevallier, L., Cohen, M., et al. 2000, A\&AS, 141, 313

Freese, K. 2000, Phys. Rep., 333, 183

Graff, D. S., Freese, K., Walker, T. P., \& Pinsonneault, M. H. 1999, ApJ, 523, L77

Hansen, B. M. S. 2001, ApJ, 558, L39

Ibata, R., Irwin, M., Bienaymé, O., Scholz, R., \& Guibert, J. 2000, ApJ, 532, L41

Knox, R. A., Hawkins, M. R. S., \& Hambly, N. C. 1999, MNRAS, 306, 736
Koopmans, L. V. E., \& Blandford, R. D. 2002, submitted to MNRAS [astro-ph/0107358]

Landolt, A. U. 1992, AJ, 104, 340

Lasker, B. M., Sturch, C. R., Lopez, C., et al. 1988, ApJS, 68, 1

Lasserre, T., Afonso, C., Albert, J. N., et al. 2000, A\&A, 355, L39

Liebert, J., Dahn, C. C., \& Monet, D. G. 1988, ApJ, 332, 891

Luyten, W. J. 1979a, LHS catalogue, A catalogue of stars with proper motions exceeding $0^{\prime \prime} 5$ annually, 2nd ed. (Minneapolis: University of Minnesota, 1979)

Luyten, W. J. 1979b, New Luyten Catalogue of stars with proper motions larger than two tenths of an arcsecond (Minneapolis: University of Minnesota)

Majewski, S. R. \& Siegel, M. H. 2002, ApJ, 569, 432

Monet, D. G., Fisher, M. D., Liebert, J., et al. 2000, AJ, 120, 1541

Nelson, C. A., Cook, K. H., Axelrod, T. S., et al. 2002, ApJ, submitted [astro-ph/0112414]

Oppenheimer, B. R., Hambly, N. C., Digby, A. P., Hodgkin, S. T., \& Saumon, D. 2001a, Science, 292, 698

Oppenheimer, B. R., Saumon, D., Hodgkin, S. T., et al. 2001b, ApJ, 550, 448

Paczynski, B., Udalski, A., Szymanski, M., et al. 1999, Acta Astron., 49, 319

Reid, I. N., Sahu, K. C., \& Hawley, S. L. 2001, ApJ, 559, 942 Reylé, C., Robin, A. C., \& Crézé, M. 2001, A\&A, 378, L53

Robin, A. C., Reylé, C., \& Crézé, M. 2000, A\&A, 359, 103 